\title{
Effect of replacing maize with sorghum, millet or 'acha' on the growth and carcass
} characteristics of broiler

${ }^{1}$ Haladu, S. and ${ }^{2}$ Sonaiya E.B

${ }^{1}$ Centre for Entrepreneurship Development, Federal Polytechnic, Ede, Osun State, Nigeria

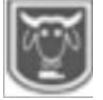

Abstract
${ }^{2}$ Department of Animal Sciences, Faculty of Agriculture,

Obafemi Awolowo University, Ile-Ife, Osun State, Nigeria, 220005

Corresponding author email: fsonaiya@gmail.com

Maize, which supplies most of the energy in poultry feeds, has low yields in Nigeria and there is competition for the limited harvest of maize between industrial, livestock and human needs. Thus, this study was carried out to evaluate the effects on the growth and carcass characteristics of broiler chickens of replacing the high inputs grain, maize, with a low inputs grain, 'acha', or medium inputs grains, sorghum and millet. Four isocaloric and isonitrogenous diets for both the starter and finisher phases were formulated with maize, sorghum, millet and 'acha' as the main source of energy and were used as treatments 1, 2, 3 and 4, respectively. Marshall broiler birds were brooded up to 2 weeks before the treatments were introduced that lasted six weeks. Up to 8 weeks of age, body weight, average daily gain, feed conversion ratio and mortality were recorded weekly. The results of the study show no significant differences $(P>0.05)$ between the four treatments in final body weight and average daily gain but birds on treatment 4 ('acha') had significantly lower feed intake and superior feed conversion ratio than the other experimental diets. It was concluded that maize can be replaced with sorghum, millet and 'acha' without adverse effects on the performance ofbroiler chickens.

Keywords: 'acha', broiler, maize, millet, sorghum

\section{Introduction}

Maize has high requirements for fertilizer and moisture when compared to sorghum, millet and acha (NAS, 1996). Maize, which supplies most of the energy in poultry feeds, has low yields (7.3 million metric tonnes) in Nigeria and there is competition for the limited harvest of maize between industrial, livestock and human needs (Oluyemi and Robert, 2000). The sudano-sahelian zone of West Africa is more conducive for sorghum, millet and acha production (Clement et al., 2010; NAS, 1996). Therefore, in order to sustain poultry production in the sudanosahelian zone, there is need to replace maize (a high inputs grain) with sorghum and millet (medium inputs grains) or acha (low inputs grain) in poultry diets.

\section{Materials and Methods}

The study was carried out at the Poultry Unit of the Teaching and Research Farm and the Poultry Meat Research Laboratory of the Department of Animal Sciences of Obafemi Awolowo University. The four grains were analysed for proximate composition according to AOAC (1990) and tested for three mycotoxins (aflatoxin fumonisin and ochratoxin) using the method of Bragulat et al. (2001). A total of 120, 2-weeks old Marshall broiler chicks were allotted into four treatments, each with three replicates of 10 birds per replicate in a randomized complete block design. Four isocaloric and isonitrogenous starter $(2800 \mathrm{kcal}, 23 \% \mathrm{CP})$ and finisher (2900 kcal, 20\% CP) diets with maize, 


\section{Maize replacement with sorghum, millet or 'acha' in broiler diets}

sorghum, millet and acha were formulated for treatments 1,2, 3 and 4, respectively. For six weeks, data were collected on feed intake, body weight, mortality and average daily gain and feed conversion ratio were calculated. The experimental diets were analysed for two anti-nutritional factors: oxalate, by the titrimetric method of Oke (1966) as modified by Falade et al. (2004), and tannin, by the modified vanillinhydrochloric acid method of Price et al. (1978). At eight weeks, three birds per treatment were randomly selected, slaughtered and dressed. Immediately after dressing, the carcasses were chilled and stored in a chest freezer $\left(-18^{0} \mathrm{C}\right)$ for three days before carcass analysis. Frozen carcasses were thawed and the abdominal fat thickness and weight were measured according to the method of Sonaiya and Benyi (1983). Carcass proportions of thigh, breast, back, neck, wings, heart, liver, and gizzard were determined. All data collected were subjected to analysis of variance and means were separated by Duncan's multiple range test.

\section{Results and Discussion}

The results of the proximate, antinutritional and mycotoxin analysis of the experimental grains obtained in this study (Table 1) are within the range reported previously (Clement et al., 2010; Ballogou et al., 2012 and Bulus et al., 2014). The compositions of experimental diets are on Tables 2 and 3. Table 4 shows the performance of broilers fed the experimental diets.

The results showed no significant differences in growth among the experimental diets. Adamu et al. (2006) reported no depressive effect on growth when sorghum completely replaced maize in poultry diets. Birds on the acha diet recorded the lowest $(\mathrm{P}<0.05)$ feed intake and superior $(\mathrm{P}<0.05)$ feed conversion ratio. This may be due to the high ME (3939 $\mathrm{kcal} / \mathrm{kg}$ ), the acha grains were on the other hand rich in methionine and cysteine, two amino acids almost deficient in the major cereals like sorghum, rice, wheat or barley as reported by Ballogou et al (2012). Birds on the sorghum diet recorded the poorest feed conversion ratio. Sorghum has the highest fibre and tannin content (Table 1) which agrees with the report of Clement (2010). Table 5 showed that there was no significant $(\mathrm{P}>0.05)$ differences in live and carcass weight among birds on the four treatments.

Table 1: Proximate, anti-nutritional and mycotoxin analyses of the experimental grains

\begin{tabular}{lllllll}
\hline Parameters & Maize & Sorghum & Millet & Acha & MEAN & SEM \\
\hline Moisture content $(\%)$ & $10.1^{\mathrm{b}} \pm 0.14$ & $9.7^{\mathrm{b}} \pm 0.42$ & $11.0^{\mathrm{a}} \pm 0.16$ & $11.0^{\mathrm{a}} \pm 0.04$ & 10.5 & 0.27 \\
Crude protein $(\%)$ & $9.3^{\mathrm{ab}} \pm 0.13$ & $10.0^{\mathrm{a}} \pm 0.20$ & $10.0^{\mathrm{a}} \pm 0.14$ & $8.0^{\mathrm{b}} \pm 0.04$ & 9.31 & 0.44 \\
Crude fibre $(\%)$ & $2.3^{\mathrm{b}} \pm 0.22$ & $4.7^{\mathrm{a}} \pm 0.25$ & $4.5^{\mathrm{a}} \pm 0.05$ & $2.1^{\mathrm{b}} \pm 0.29$ & 3.4 & 0.15 \\
Ether extract $(\%)$ & $4.5^{\mathrm{b}} \pm 0.74$ & $2.7^{\mathrm{a}} \pm 0.35$ & $5.0^{\mathrm{a}} \pm 0.32$ & $1.6^{\mathrm{c}} \pm 0.12$ & 3.2 & 0.44 \\
Ash $(\%)$ & $2.2^{\mathrm{c}} \pm 0.7$ & $2.6^{\mathrm{bc}} \pm 0.06$ & $3.0^{\mathrm{a}} \pm 0.13$ & $2.7^{\mathrm{ab}} \pm 0.04$ & 2.6 & 0.13 \\
Nitrogen free extract & $71.6 \pm 0.03$ & $70.3 \pm 0.88$ & $66.5 \pm 0.52$ & $74.6 \pm 0.74$ & 71.2 & 0.82 \\
Tannin $(\mathrm{mg} / \mathrm{g})$ & $0.3^{\mathrm{b}} \pm 0.2$ & $3.1^{\mathrm{a}} \pm 2.2$ & $0.6^{\mathrm{b}} \pm 0.4$ & $0.9^{\mathrm{b}} \pm 0.1$ & 1.2 & 1.06 \\
Oxalate $(\mathrm{mg} / 100 \mathrm{~g})$ & $18^{\mathrm{a}} \pm 0.6$ & $11^{\mathrm{c}} \pm 0.3$ & $18^{\mathrm{a}} \pm 0.4$ & $15.6^{\mathrm{b}} \pm 0.3$ & 15.8 & 0.50 \\
Aflatoxin $(\mu \mathrm{g} / 100 \mathrm{~g})$ & $0.80^{\mathrm{a}}$ & $0.93^{\mathrm{a}}$ & $0.03^{\mathrm{b}}$ & $0.02^{\mathrm{b}}$ & 0.4 & 0.12 \\
Ochratoxin $(\mu \mathrm{g} / 100 \mathrm{~g})$ & $0.01^{\mathrm{b}}$ & $1.50^{\mathrm{a}}$ & $0.04^{\mathrm{b}}$ & $0.01^{\mathrm{b}}$ & 0.4 & 0.05 \\
Fuminosin $(\mathrm{mg} / \mathrm{kg})$ & $0.01^{\mathrm{c}}$ & $7.83^{\mathrm{a}}$ & $0.01^{\mathrm{c}}$ & $3.00^{\mathrm{b}}$ & 2.7 & 0.14 \\
\hline
\end{tabular}

Means in the same row having different superscripts differ significantly at $\mathrm{P}<0.05$. 
Haladu and Sonaiya

Table 2: Composition of different energy grains-based diets for starter broiler chickens

\begin{tabular}{|c|c|c|c|c|}
\hline \multicolumn{5}{|c|}{ Experimental diets } \\
\hline Ingredients & $\begin{array}{l}\text { Treatment } 1 \\
\text { (Maize) }\end{array}$ & $\begin{array}{l}\text { Treatment } 2 \\
\text { (Sorghum) }\end{array}$ & $\begin{array}{l}\text { Treatment } 3 \\
\text { (Millet) }\end{array}$ & $\begin{array}{l}\text { Treatment } 4 \\
\text { (Acha) }\end{array}$ \\
\hline Test grain & 50.00 & 52.00 & 53.00 & 45.00 \\
\hline Soybean meal & 34.60 & 34.00 & 33.00 & 32.00 \\
\hline Wheat offal & 6.00 & 5.00 & 5.00 & 5.00 \\
\hline Palm kernel cake & 2.00 & 2.00 & 2.00 & 3.00 \\
\hline Fish meal & 3.00 & 3.00 & 4.00 & 6.50 \\
\hline Bone meal & 3.00 & 2.85 & 2.00 & 4.90 \\
\hline Limestone & 0.50 & 0.50 & 0.40 & 3.00 \\
\hline Vit. Premix & 0.45 & 0.20 & 0.20 & 0.20 \\
\hline Common salt & 0.25 & 0.20 & 0.20 & 0.20 \\
\hline Methionine & 0.15 & 0.20 & 0.10 & 0.10 \\
\hline Lysine & 0.05 & 0.05 & 0.10 & 0.10 \\
\hline Calculated values (\%) & 100 & 100 & 100 & 100 \\
\hline ME (kcal/kg) & 2893 & 2856 & 2880 & 2884 \\
\hline Crude protein & 23.07 & 23.37 & 23.25 & 23.11 \\
\hline Crude fibre & 4.02 & 3.95 & 4.97 & 3.48 \\
\hline Ether extract & 3.68 & 3.70 & 3.75 & 2.71 \\
\hline Calcium & 1.55 & 1.49 & 1.21 & 3.34 \\
\hline Phosphorus & 0.81 & 0.79 & 0.69 & 1.22 \\
\hline Lysine & 1.55 & 1.32 & 1.47 & 1.43 \\
\hline Methionine & 0.52 & 0.57 & 0.48 & 0.58 \\
\hline \multicolumn{5}{|c|}{$\begin{array}{l}\text { Premix provided with chicks: vitamin } A: 1000 \mathrm{IU} \text {; vitamin } D_{3}: 500 \mathrm{IU} \text {; vitamin } \mathrm{E}: 5.75 \mathrm{IU} \text {; vitamin } \mathrm{K}_{3} \text { : } \\
0.5 \mathrm{mg} \text {; vitamin } B_{1}: 0.45 \mathrm{mg} \text {; vitamin } B_{2}: 1.25 \mathrm{mg} \text {; vitamin } B_{6}: 0.75 \mathrm{mg} \text {; vitamin } B_{12}: 0.00375 \mathrm{mg} \text {; } \\
\text { Niacin: } 6.875 \mathrm{mg} \text {; Pantothenic acid: } 1.875 \mathrm{mg} \text {; Folic acid } 0.1875 \mathrm{mg} \text {; Biotin } \mathrm{H}_{2}: 0.015 \mathrm{mg} \text {; C Choline }\end{array}$} \\
\hline
\end{tabular}


Maize replacement with sorghum, millet or 'acha' in broiler diets

Table 3: Composition of different energy grains-based diets for finisher broiler chickens

\begin{tabular}{lllll}
\hline \multicolumn{5}{c}{ Experimental diets } \\
\hline Ingredients & $\begin{array}{l}\text { Treatment 1 } \\
\text { (Maize) }\end{array}$ & $\begin{array}{l}\text { Treatment } \\
\text { (Sorghum) }\end{array}$ & $\begin{array}{l}\text { Treatment 3 } \\
\text { (Millet) }\end{array}$ & $\begin{array}{l}\text { Treatment 4 } \\
\text { (Acha) }\end{array}$ \\
\hline Test grains & 60.00 & 62.00 & 62.00 & 54.00 \\
Soybeans meal & 25.00 & 25.00 & 25.40 & 22.40 \\
Wheat Offal & 5.45 & 5.00 & 4.00 & 4.00 \\
Palm Kernel Cake & 2.00 & 2.00 & 2.00 & 4.00 \\
Fish meal & 3.40 & 3.00 & 3.25 & 7.00 \\
Bone meal & 3.00 & 2.00 & 2.00 & 3.00 \\
Limestone & 0.50 & 0.45 & 0.50 & 3.00 \\
Vitamin/ mineral premix & 0.25 & 0.20 & 0.25 & 2.00 \\
Common salt & 0.25 & 0.20 & 0.20 & 0.20 \\
Methionine & 0.10 & 0.10 & 0.20 & 0.20 \\
Lysine & 0.05 & 0.05 & 0.20 & 0.20 \\
Total (\%) & $\mathbf{1 0 0}$ & $\mathbf{1 0 0}$ & $\mathbf{1 0 0}$ & $\mathbf{1 0 0}$ \\
Calculated values & & & & \\
Metabolizable energy & 2978 & 2943 & 2930 & 2977 \\
Kcal/kg & & & & \\
Crude protein (\%) & 20.23 & 20.69 & 20.24 & 20.17 \\
Crude fibre (\%) & 3.56 & 3.56 & 4.74 & 3.00 \\
Ether extract (\%) & 3.74 & 3.17 & 3.78 & 2.61 \\
Calcium (\%) & 1.56 & 1.16 & 1.18 & 2.65 \\
Available phosphorus (\%) & 0.77 & 0.76 & 0.63 & 0.90 \\
Lysine (\%) & 1.11 & 1.15 & 1.34 & 1.29 \\
Methionine (\%) & 0.43 & 0.38 & 0.53 & 0.66 \\
\hline
\end{tabular}

Premix provided to chicks with: vitamin A: $1000 \mathrm{IU}$; vitamin $D_{3}$ : $500 \mathrm{IU}$; vitamin E: $5.75 \mathrm{IU}$; vitamin $K_{3}: 0.5 \mathrm{mg}$; vitamin $B_{1}: 0.45 \mathrm{mg}$; vitamin $B_{2}: 1.25 \mathrm{mg}$; vitamin $B_{6}: 0.75 \mathrm{mg}$; vitamin $B_{12}: 0.00375 \mathrm{mg}$; Niacin: $6.875 \mathrm{mg}$; Pantothenic acid: $1.875 \mathrm{mg}$; Folic acid $0.1875 \mathrm{mg}$; Biotin $\mathrm{H}_{2}: 0.015 \mathrm{mg}$; C Choline chloride: $75 \mathrm{mg}$; Cobalt: $0.05 \mathrm{mg}$; Copper: $0.75 \mathrm{mg}$; Iodine: $0.25 \mathrm{mg}$; Iron: $5 \mathrm{mg}$; Manganese: $10 \mathrm{mg}$; Selenium: $0.05 \mathrm{mg}$; Zinc: $7.5 \mathrm{mg}$ and Antioxidant: $0.3125 \mathrm{mg}$

Table 4: Performance of broilers fed different energy grains-based experimental diets

\begin{tabular}{lllllll}
\hline Parameters & $\begin{array}{c}\text { Experimental Diets } \\
\text { Treatment 1 } \\
\text { (Maize) }\end{array}$ & $\begin{array}{l}\text { Treatment 2 } \\
\text { (Sorghum) }\end{array}$ & $\begin{array}{l}\text { Treatment 3 } \\
\text { (Millet) }\end{array}$ & $\begin{array}{l}\text { Treatment 4 } \\
\text { (Acha) }\end{array}$ & Mean & SEM \\
\hline $\mathrm{BW}(2$ wks), g & $223 \pm 6$ & $217 \pm 2$ & $218 \pm 1$ & $218 \pm 1.7$ & 219.5 & 3.8 \\
$\mathrm{BW}(8$ wks), g & $1481 \pm 103$ & $1297 \pm 68$ & $1428 \pm 153$ & $1449 \pm 87$ & 1414.0 & 107 \\
$\mathrm{ADG}, \mathrm{g}$ & $30.0 \pm 2.56$ & $25.7 \pm 1.67$ & $28.8 \pm 3.64$ & $29.3 \pm 2.28$ & 28.5 & 2.6 \\
$\mathrm{ADFI}, \mathrm{g}$ & $96.6^{\mathrm{a}} \pm 0.85$ & $92.0^{\mathrm{a}} \pm 5.04$ & $93.4^{\mathrm{a}} \pm 5.72$ & $77.2^{\mathrm{b}} \pm 10.90$ & 89.8 & 4.8 \\
FCR & $3.2^{\mathrm{a} b} \pm 0.30$ & $3.6^{\mathrm{a}} \pm 0.10$ & $3.3^{\mathrm{ab}} \pm 0.60$ & $2.7^{\mathrm{b}} \pm 0.57$ & 3.2 & 0.37 \\
Mortality $(\%)$ & $3.0^{\mathrm{b}} \pm 5.77$ & $13.0^{\mathrm{a}} \pm 5.77$ & $20.0^{\mathrm{a}} \pm 0.00$ & $13.0^{\mathrm{ab}} \pm 11.55$ & 12.3 & 5.8 \\
\hline
\end{tabular}

${ }^{\mathrm{ab}}$ Means in the same row having different superscripts differ significantly at $\mathrm{P}<0.05$.

BW (2 wks): Body weight at two weeks, BW( 8 wks): Body weight at eight weeks, ADG: Average daily gain, ADFI: Average daily feed intake, FCR: Feed conversion ratio. 


\section{Haladu and Sonaiya}

Table 5: Carcass characteristics of broilers fed different energy grains-based experimental diets

\begin{tabular}{|c|c|c|c|c|c|c|}
\hline \multirow[b]{2}{*}{ Parameters } & \multicolumn{4}{|c|}{ Experimental Diets } & \multirow[b]{2}{*}{ Mean } & \multirow[b]{2}{*}{ SEM } \\
\hline & $\mathbf{1}$ & 2 & 3 & 4 & & \\
\hline LW (g/bird) & $1533 \pm 124$ & $1323 \pm 108$ & $1397 \pm 205$ & $1456 \pm 19$ & 1427.8 & 107.54 \\
\hline WCW (g/bird) & $1291 \pm 107$ & $1119 \pm 112$ & $1165 \pm 170$ & $1218 \pm 46$ & 1198.6 & 95.50 \\
\hline Fat thickness (mm) & $4.3 \pm 0.53$ & $2.3 \pm 0.58$ & $3.7 \pm 2.08$ & $2.3 \pm 0.58$ & 3.2 & 1.04 \\
\hline Fat weight $(\%)$ & $1.3 \pm 0.06$ & $1.1 \pm 0.51$ & $1.0 \pm 0.46$ & $0.8 \pm 0.13$ & 1.04 & 0.38 \\
\hline Thigh weight (\%) & $21.0 \pm 0.93$ & $21.1 \pm 0.59$ & $23.1 \pm 1.83$ & $21.2 \pm 1.35$ & 21.61 & 1.42 \\
\hline Breast weight (\%) & $16.1^{\mathrm{ab}} \pm 1.45$ & $14.6^{\mathrm{b}} \pm 0.28$ & $15.3^{\mathrm{ab}} \pm 1.01$ & $17.3^{\mathrm{a}} \pm 1.38$ & 15.81 & 1.17 \\
\hline Wing $(\%)$ & $7.8^{\mathrm{b}} \pm 0.45$ & $8.8^{\mathrm{a}} \pm 0.21$ & $8.5^{\mathrm{ab}} \pm 0.49$ & $9.2^{\mathrm{a}} \pm 0.53$ & 8.59 & 0.45 \\
\hline $\operatorname{Neck}(\%)$ & $5.6 \pm 0.31$ & $5.5 \pm 0.56$ & $5.6 \pm 0.47$ & $4.9 \pm 0.43$ & 5.42 & 0.51 \\
\hline Heart $(\%)$ & $0.56 \pm 0.06$ & $0.48 \pm 0.07$ & $0.53 \pm 0.07$ & $0.54 \pm 0.06$ & 0.53 & 0.07 \\
\hline Liver $(\%)$ & $2.5 \pm 0.52$ & $2.4 \pm 0.18$ & $2.5 \pm 0.18$ & $2.9 \pm 0.28$ & 2.61 & 0.33 \\
\hline Gizzard (\%) & $2.3 \pm 0.18$ & $2.4 \pm 0.52$ & $2.2 \pm 0.49$ & $1.9 \pm 0.14$ & 2.22 & 0.34 \\
\hline
\end{tabular}

There was no significant difference among the treatments in all the carcass parameters except breast weight. Rama Rao et al. (2001) and Reddy et al. (2008) reported no significant difference in carcass characteristics when maize was replaced in poultry diets with sorghum at $100 \%$ or $50 \%$, respectively. Bulus et al. (2014) reported a significant decrease in carcass measures when maize was totally replaced by millet in poultry diets. In this study, higher breast weight was observed in birds fed the acha diet as compared with those fed the maize diet. This agrees with the report of Ukim et al. (2012).

\section{Conclusion}

The findings of this study showed that maize can successfully be replaced with sorghum, millet or acha without adverse effects on performance of broiler chickens.

\section{Acknowledgement}

The support of the African Chicken Genetic Gains, Nigeria (ACCG-NG) project in paying for this publication is appreciated

\section{References}

Association of Official Analytical Chemistry AOAC, 1990. Official Method of Analysis, 15th Edn. Washington D.C., USA.

Adamu, M. S., Nafarnda, W. D., Iliya, D. S. and Kubkomawa, H. I., 2006. Replacement value of yellow sorghum (Sorghum bicolor) for maize in broiler diets. Global Journal of Agricultural Science, 5(2): 151-154.

Ballogou, V.Y., Soumanou, M.M., Toukourou, F. and Hounhouigan, J.D., 2013. Structure and nutritional composition of fonio (Digitaria exilis) grains: A review. Intern. Res. J. of Bio. Sci. Vol. 2(1): 73-79

Bragulat, M. R., Abarca, M. L. and Calanes F. J., 2001. An easy screening method for fungi producing ochratoxin-A in pure culture. International Journal of foodmicrobiology 71:139-144.

Bulus, E. D., Ibe, E. A., Dodo, S. T., Samuel, I. and Makinde, O. J., 2014. Performance of broiler chickens fed two varieties of 


\section{Maize replacement with sorghum, millet or 'acha' in broiler diets}

guinea corn and millets as replacement for maize. Iranian Journal of Applied Animal Science, 4(3): 541-547.

Clement I. M., Ibrahim D. K., Joseph I., Iro N., Ibrahim D. M., and Bruce H., 2010. Performance and economics of production of broiler chickens fed sorghum or millet as replacement for maize in the semiarid zone of Nigeria. Agriculture and Biology Journal of North America, ISSN Online: 2151-7525

Clement I. M., Ibrahim D. K., Joseph I., Iro N., Ibrahim D. M., and Bruce H., 2010. Performance and economics of production of broiler chickens fed sorghum or millet as replacement for maize in the semiarid zone of Nigeria. Agriculture and Biology Journal of North America, ISSN Online: 2151-7525

Falade, O. S., Dare A. F., Bello, M. O., Osuntogun, B. O., and Adewusi, S. R. A., 2004. Varietal Changes in Proximate Composition and the Effect of Processing on the Ascorbic acid Content of some Vegetables. Journal food Technology 2:103-108.43.

National Academy of Science, 1996. "Fonio (Acha)". Lost Crops of Africa: Volume I: Grains. Lost Crops of Africa, 1. National Academies Press. ISBN 978-0$\begin{array}{lllllllllll}3 & 0 & 9 & - & 0 & 4 & 9 & 9 & 0 & - & 0\end{array}$. http://books.nap.edu/openbook.ph p record_id $=2305 \&$ page $=59$. Retrieved 14th October, 2015.
Oke, O. L., 1969. Oxalic Acid in Plants and in Nutrition. World Review of Nutrition and Dietetics, 10: 263303.

Price, M. L., Scoyoc, S. V., and Butler, L. G., 1978. A Critical Evaluation of the Vanillin Reaction as an Assay for Tannin in Sorghum Grain. Journal of Agriculture and Food Chemistry 26: 1214-1218.

Rama Rao, S. V; Shyam Sunder, G. I; Reddy, M. R; Praharaj, N. K; Raju, M. V. and Panda, A. K., 2001. Effect of supplementary choline on the performance of broiler breeders fed on different energy sources. Journal of British Poultry Science, 42: 362-367.

Reddy V. K., Malathi, V., Venkatarami, B. S., Reddy, K., Pratap Kumar, S., and Jayanaik, B., 2008. Effect of finger millet and sorghum replacing corn in presence of soy oil/ fish oil and enzymes on performance of broilers. International Journal of Poultry Science, 7 (6): 560-546.

Sonaiya E. B. and Benyi K., 1982. Abdominal fat in 12 to 16 week-old broiler birds as influenced by age, sex, and strain. Br. Poultry. Sci. 62:1793-1799.

Ukim, C. I., Ojewola G. S., Obun, C. O. and Ndelekwute, E. N., 2012. Perfomance, carcass and organs weight of broiler chicks fed graded levels of acha grains (Digitaria exilis). Journal of Agriculture and Vet.Sci. 1(1): 28-33.

Received: 287h October, 2017 Accepted: 30th November, 2017 